\title{
Improvement of Image Deblurring Through Different Methods
}

\author{
A. Anusha ${ }^{1}$, Dr. P. Govardhan ${ }^{2}$. \\ ${ }^{1}$ computer Science /Vardhaman College Of Engineering /India \\ ${ }^{2}$ information Technology/Vardhaman College Of Engineering /India
}

\begin{abstract}
In this paper, we analyze the research on this topic both theoretically and experimentally through different methods the deterministic filter; Bayesian estimation, the conjunctive deblurring algorithm (CODA), and alpha tonal correction method which performs the deterministic filter and Bayesian estimation in in a conjunctive manner. We point out the weakness of the deterministic filter and unify the limitation latent in two kinds of Bayesian estimators. .The proposed algorithm, alpha tonal correction methods, which gives better performance than the deterministic filter and sharp image estimation. We point out the weaknesses of the deterministic filter and unify the limitation latent in two kinds of image estimation methods. I further explain proposed alpha correction method which can able to handle quite large blurs beyond deterministic filter and image estimation. Finally, I demonstrate that our method outperforms state-of-the-art methods with a large margin.
\end{abstract}

Key words -Blind image DE convolution, image sharpening, alpha Tonal correction and deterministic filter.

\section{Introduction}

Recovery of a sharp image from a blurred one is a chronic ill proposed problem for many scientific applications, such as astronomical Imaging and consumer photography. Generally, there are many properties of a camera and a scene that can lead to blur, i.e., spatially uniform defocus blur dependent on depth, spatially varying defocus blur due to focal length variation over the image plane, spatially uniform blur due to camera translation, spatially varying blur due to camera roll, yaw and pitch motions, and spatially varying blur due to object movements. In this paper, Our goal is to reveal the limitations and potentials of recent methods when dealing with quite large blurs and severe noise. What are the main challenges and what are the key components that make handling quite large blurs and severe noise possible? What should attract further research efforts in the future additionally; additionally we design a novel deblurring method to handle various large blurs and significant noise. I consider the research on this topic has evolved mainly through two paradigms

1) The deterministic sharpening filter 2)sharpening image Bayesian estimation using blind DE convolution method. in this paper I focus on third paradigm the alpha tonal correction method in next review these Three paradigms by reveling the latent limitations.

\section{.Alpha Rooting}

Alpha rooting is a transform based enhancement algorithm [7]. It works by performing some orthogonal transform, such as the Fourier Transform; modifying the coefficients with the phase kept invariant, and performing the inverse transform. The coefficient magnitudes are modified as follows:

$$
O(p, s)=X(p, s) \times|X(p, s)|^{\alpha-1}
$$

\section{Different Deblurring Techniques} denoting

The deterministic filter can be modeled as deterministic func-tionof the input blurred image, with

The output sharp image. The leftmost flowchart in Fig. 1illustrates the first paradigm. One of the most well-known approachesin this paradigm is unsharp masking, of which the basic idea is to reduce the low frequency first, and then highlightthe high-frequency components. The performance varies according to the adopted high-pass filters and the adaptive edge weights 


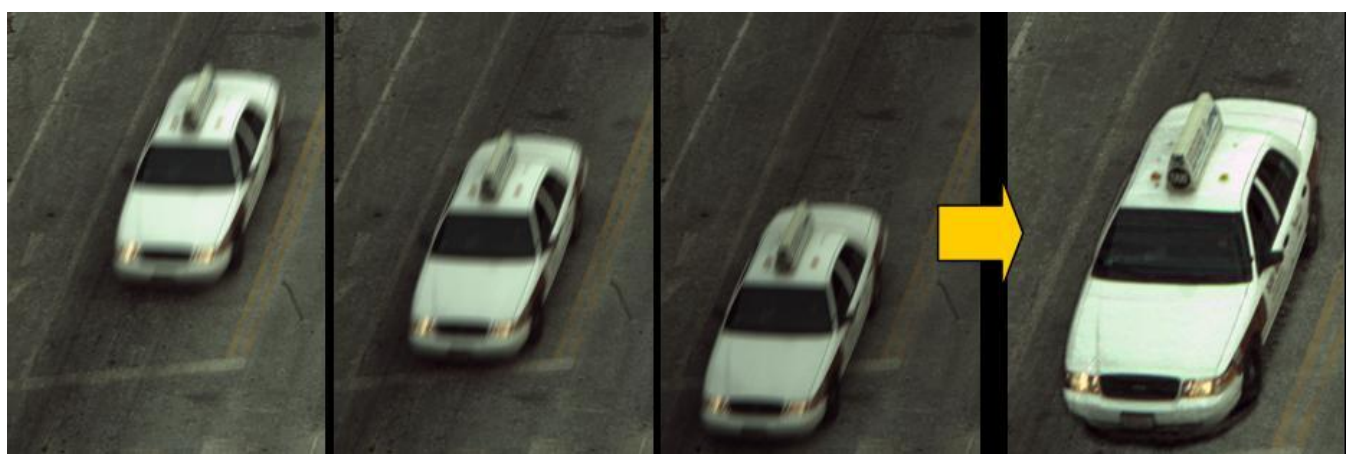

Fig.1 Example for Image deblurring

This approach assumes that the blurred edges do not drift too far away from the latent sharp edges; thus, it can handle only the defocus blurs and very small motion blurs. For very large blurs, the image narrow edges or details are severely damaged and very difficult to restore. Apractical solutionis to detect and restore large step edges explicitly or implicitly,which we call the step-edge-based filter (SEBF). Explicit SEBFfirst locates the step edge and then propagates the local intensity extrema toward the edge. Implicit SEBF performs edge detection and restoration in a single step, based on zero crossings of high-pass filters. Commonly used implicit SEBFs include the shock filter, the backward diffusion, the morphologica lfiltering, the fuzzy operator and many other adapted versions. Compared with the second paradigm, i.e., Bayesian estimation, the SEBF has the following advantages: 1) The SEBF can handle various blurs without adaptation because it is independent of the blurring processes (blur models), and 2) The per-formance of the SEBF is not constrained by the sample number(SN) because it depends on image local features rather than sufficient samples. The first advantage has been noticed by Joshi et al. They propose a novel SEBF to handle complex but small natural blurs.

\subsection{Limitations of the first paradigm}

The deterministic filter have been widely used in sharpening small blurs. The SEBF can handle very large blur kernels. We take the shock filter as an example, i.e.

$I^{(t+1)}=I^{(t)}-\operatorname{sign}\left(\Delta I^{(t)}\left\|\nabla I^{(t)}\right\|_{1} d t\right)$

where $I^{(t)}$ is an image at time , $\Delta$ is Laplacian operator, and $d t$ is the time step for a single evolution. The shock filter sharpens image at infection points (zero crossings of the second derivative), thus depending on image local features rather the SN.The local extreme of remain unchanged in the evolutions. Fig. 2 gives two small images blurred by large kernels. Although it is intuitively correct that Bayesian estimation can handle most blurred images, experiments of the aforementioned MAP estimators have shown that the performance is not always stable, sometimes even worse than the deterministic filters. The unstable performance gain is due to the following reasons:

1) A Bayesian estimator is built for a specific blur model and cannot handle other types of blurs without adaptation.

2) The performance highly depends on the SNs and statistics.

\subsection{Bayesian Estimation}

In this paradigm, both the kernel and image are taken as samples from some probability spaces. The goal is to solve for the unknowns that minimize the expected value of a loss function. The most commonly used loss function is the Dirac delta function, which yields the maximum a posterior (MAP) estimator. The center flow chart in Fig.1shows such a second-paradigm approach. Bayesian estimation has been recently hotly discussed because it has led to great progress. The success of it stems from the use of various image priors and estimators. In the MAP (L, K) case, which solves for both the kernel and image simultaneously, and a MAP (L, $\mathrm{K})$ case, which solves for the kernel alone. It has been pointed out that naive a MAP (L,K) estimator fails to yield the desired result since the sparse priors prefer no-blur explanations. Current MAP estimators avoid the trivial solution by integrating many additional components, such as sharp edge detection iterative likelihood update and sparse representation under frame let and curve let system. By contrast, the MAP (L, K) estimator is well constrained and can accurately recover the true kernel if the image size is much arger than the kernel size Compared. With the first paradigm, Bayesian estimation has the following advantages:

1) The approach is not sensitive to local narrow edges because it depends on statistics,

2) It is not sensitive to image noise if the noise is not too much to change the statistics. 


\section{Limitations of Bayesian Estimation}

Fig. 2 demonstrates that the MAP and MAP estimators have very similar performance with respect to the same SN. They both belong to Bayesian estimation and should have similar properties. The estimation states that theory states that when the SN is small, the Bayesian estimation will be "biased" toward the prior mean, which, however, is not the true solution in the blind de-convolution case. From the perspective of the energy function to understand this limitation.

\subsection{Blind deconvolution in image processing}

In image processing and applied mathematics, blind deconvolution is a deconvolution technique that permits recovery of the target scene from a single or set of "blurred" images in the presence of a poorly determined or unknown point spread function (PSF). Regular linear and non-linear deconvolution techniques utilize a known PSF. For blind deconvolution, the PSF is estimated from the image or image set, allowing the deconvolution to be performed. Researchers have been studying blind deconvolution methods for several decades, and have approached the problem from different directions

\section{Alpha Rooting}

Alpha rooting is a transform based enhancement algorithm [7]. It works by performing some orthogonal transform, such as the Fourier Transform; modifying the coefficients with the phase kept invariant, and performing the inverse transform. The coefficient magnitudes are modified as follows:

$$
O(p, s)=X(p, s) \times|X(p, s)|^{\alpha-1}
$$

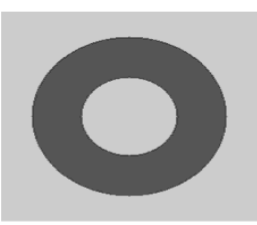

(a)

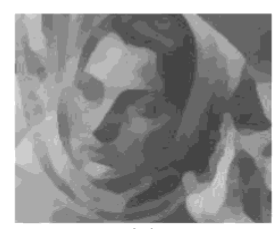

(f)

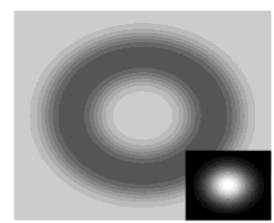

(b)

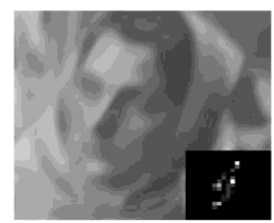

$(\mathrm{g})$

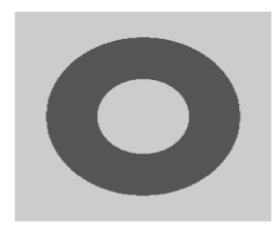

(c)

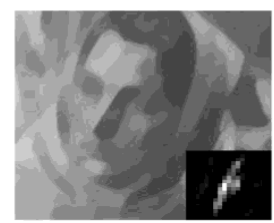

(h)

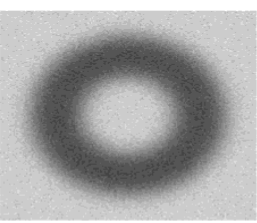

(d)

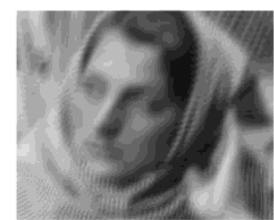

(i)

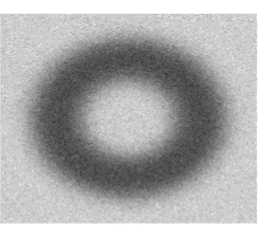

(e)

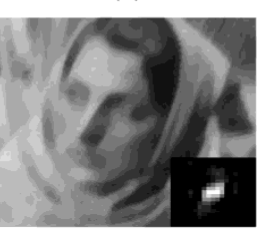

(j)

Fig.2. (a)Original sharp image. (b) The blurred image without noise. The Gaussian kernel is producedby MATLAB with special ("Gaussian", 61, 11). (c) The recovered image from (b) by the shock filter. (d) The blurred image with large noise. (e) The recovered image from (d) by the shock filter. (f) The original sharp image without narrow edges. (g) The blurred image and the kernel. (h) The recovered image from $(\mathrm{g})$ by the shockk filter. PSNR

Where $O(p, s)$ is the 2-D Fourier Transform of the output image, $X(p, s)$ is the 2-D Fourier transform of the input image, and the range of alpha is $0=a=1$. As alpha rooting reduces the magnitude of the transform coefficients, it naturally compresses the range of an image. Also, as the phase data remains unchanged, image edges are preserved. Because of this, it is understandable that this would be used to process the smoothed image.

Figure 5 shows sample results for the method in figure 2, using alpha rooting to process the smoothed image. These resulting images have good contrast and show the important image objects without sacrificing detail.The goal of image enhancement is to improve the image quality of the de-blurred image so that the resultant image is better than the original image for a specific application or set of objectives. Proposed are new contrast measure and novel image enhancement algorithms. The contrast measure is derived from Michelson's law of the human visual system and is used in spatial domain. Proposed algorithms performances are quantitatively compared the one of the best transform based image enhancement algorithm: $\boldsymbol{\alpha}$-rooting. The fundamental advantages of these algorithms are

a) They perform "better" than modified $\alpha$-rooting

b) They can be used for enhancement of images in the decompression stage.

c) They can be used for automatically choosing the best enhancement method, and the best parameters

A simple multi-scale image enhancement algorithm for compressing image dynamics and enhancing image details in the Fourier transform domain is presented. First, an image is separated into illumination and reflectance components. Next, the illumination component is manipulated adaptively for image dynamics by using a content measure. The content measure using the energy distribution of the coefficients is defined directly 
in each block of an image. Then, the reflectance component is altered by a multi-scale $4 \alpha$-rooting method for enhancing image details based on human visual perception. The main advantage of the proposed algorithm enhances the details in the dark and the bright areas with low computations without boosting noise information and affecting the compressibility of the original image since it performs on the images in the compressed domain. In order to evaluate the proposed scheme, several base-line approaches can be described and compared using enhancement quality measures.

\section{1alpha Tonal Correction}

The adaptive tonal correction algorithm presented here uses the low- exposure or darker looking image as its input and enhances its appearance via tonal correction by making use of the mean (brightness) and variance (contrast) of the original blurred image in an adaptive manner. The main contribution here thus consists of an automatic process by which the tonal correction is done. The following tonal curve equation is considered in our algorithm is:

$$
f(X)=\frac{\log (a x-x+1)}{\log x}
$$

Whereas $\mathrm{x}$ denotes pixel values of the input image, and the $\alpha$ is a parameter altering the brightness $\alpha$ s level. The optimal value of $\alpha$ is considered to be the one that makes the brightness of the enhanced image equal to the brightness of the blurred image This correction also improves the image contrast. To further improve the contrast, a second tonal correction curve can be used to match the contrast of the blurred image. Among various possible curve functions.

$$
g(x)=\frac{\arctan (\beta(f(x)-0.5))+0.5)}{2 \tan (\beta / 2)}-(3)
$$

Whereas $\beta$ a parameter altering theContrast level. The optimum value of $\beta$ is taken to be the one that makes the contrast of the enhanced image equal to the contrast of the blurred image.To obtain the optimum parameter values in a computationally efficient manner, the binary search approach is used.

\section{Conclusion}

Recovery of the sharp image from a blurred one is an important and long-standing problem for many applications. In this paper, we have rest analyzed the potentials and limitations latent in recent methods when handling quite large blurs and significant noise. While our method.out performs state-ofthe-art methods both in robustness to noise and the capability of handling quite large blurs, it is still limited by the images dominated by narrow edges. Recovering the totally damaged narrow edges is still a very challenging problem faced by state-of-The-art methods and should attract further research methods.

\section{References}

[1]. Kundur andD.Hatzinakos, "Blind image deconvolution,”IEEESignal Process. Mag., vol. 13, no. 3, pp. 43-64, May 1996.

[2]. J. Cai, H. Ji, C. Liu, and Z. Shen, "Blind motion deblurring from a single image using sparse approximation," inProc.IEEEConf.Comput.Vis.Pattern Recog., 2009, pp. 104-111.

[3]. N.Joshi, R.Szeliski,andD.Kriegman,"PSFestimationusingsharpedgeprediction,"inProc.IEEEConf. Comput. Vis. Pattern Recog., 2008

[4]. Gilboa, N. Sochen, and Y. Y. Zeevi“Forward-and-backward diffusion Processes foradaptive image enhancement and denoising," IEEETrans. Image Process., vol. 11, no. 7, pp.689-703, Jul. 2002

[5]. Y.Zhang, C.W e n, and Y.Zhang "estimation of motion parametersFrom blurred images"Pattern recognitionLetters.vol 21 p, pp. 425433,2000

[6]. R. Fergus, B. Singh, A. Hertzmann, S. T.Roweis, and W. T. Freeman, "Removing camera shake from a single photograph," ACM Trans. Graph., vol. 25, no. 3, pp.787-794, Jul. 2006. 